\title{
Comparative study of Metallic and Polymer Composite Shells for Underwater Vessels Using FEA
}

\author{
Moorthy Govindaraj ${ }^{{ }^{*} \text {, }}$ Narasimha Murthy Heddale Narayanarao ${ }^{1}$, Krishna \\ Munishaiah ${ }^{1}$ and Raghavendra Nagappa ${ }^{1}$ \\ ${ }^{I}$ Department of Mechanical Engineering, R.V.College of Engineering, Bangalore, India
}

(Manuscript Received June 25 2013; Revised July 18, 2013; Accepted August 27, 2013)

\begin{abstract}
The present research was aimed at comparing performance of metallic and polymer composite shells of a typical underwater vessel of length and inner diameter of $1650 \mathrm{~mm}$ and $350 \mathrm{~mm}$ respectively, based on the critical buckling pressure for operating depth of $1000 \mathrm{~m}$ using ANSYS. High strength steel, aluminium alloy, titanium alloy, glass / epoxy and carbon / epoxy materials were examined. The results indicated weight savings of $46 \%$ in carbon/epoxy and $31 \%$ in glass / epoxy when compared with high strength steel, based on the thickness of the shell for sustaining $10 \mathrm{MPa}$ buckling pressure.
\end{abstract}

Keywords: Critical buckling pressure, Underwater vessels, Polymer composite shells, ANSYS

\section{Introduction}

Underwater vessels suffer buckling due to hydrostatic pressure which reduces their load carrying capacity. Application of polymer composites for underwater vehicles can reduce their weight and expand the depth of operation. Materials for underwater vessels must not only be capable of withstanding very high external pressures, but also have other properties such as good resistance to corrosion, high strength to weight ratio, good sound absorption qualities, formability, operating life span of the material and the like. An advantage of using fibre-reinforced composites over conventional materials is that they can be tailored to the requirements of a certain applications.

Alternate materials especially polymer composites for underwater vessels have been examined in the recent research. Ross [1] proposed glass/polyester for underwater vehicles based on the influence of

\footnotetext{
*Corresponding author. Tel.: +00 please fill in, Fax.: +00 please fill in,

E-mail address: moorthyg2000@gmail.com

Copyright (C) KSOE 2013.
}

operating environment and material properties on their structural stability. Joung et al. [2] used CFRP/GFRP to design an unmanned underwater vehicle. Along with external pressure tests the cylinders were modelled using FEM like ANSYS, MSC/NASTRAN to correlate the test results. Experiments on submarine structures using cylinders, spheres and half spheres were conducted by Carvelli et al. [3] using GFRP, in undersea environment. Genetic algorithm was tried to optimise the buckling capacity of thin underwater composite cylindrical vessels by Massager et al. [4] which gave an optimum laminae stacking fibre orientation of angle which include $\left[30^{\circ}, 45^{\circ}, 60^{\circ}, 75^{\circ}, 90^{\circ}\right]$ and compared with $\pm 55^{\circ}$ orientation for carbon and glass fiber with epoxy resin and the author also correlated with experimental data. Ng RKH et al. [5] contributed to testing vessels under shallow external pressure using e-glass/epoxy woven composite material for a semi-autonomous underwater vehicle and compared with numerical analysis. Derek Graham [6] tested small scale submarines vessels and analysed the real scale submarines us- 
ing experimental data for deep sea oceans with application of polymer composite material. Hahn et al. [7] suggested that the filament winding should be the manufacturing method for underwater vehicle and the authors suggested that filament winding manufacturing method influences the buckling capacity with hydrostatic compressive external loading. Hernandez-Moreno et al. [8] related the mechanical strength to the filament winding pattern of glass/epoxy cylinders exposed to external pressure and series of implosion tests was carried out in a hyperbaric chamber, on cylindrical specimens of two pattern sizes and two wall thicknesses, made of continuous glass roving and epoxy resin. Geier et al. [9] showed that, prepeg laminations made the buckling capacity depend more on stacking order and fibre orientations. The buckling and failure characteristics of moderately thick-walled filamentwound carbon-epoxy composite cylinders under external hydrostatic pressure were simulated using commercial codes and this lead to the explanation of the fact that why cylinders collapse after buckling without recovering the initial buckling pressure. Major failure modes were directly linked to the Chul-Jin Moon et al [10] simulated using ACOS , an in-house code which was later compared with commercial codes, MSC.NASTRAN and MSC.MARC, for comparison of the buckling pressure and mode shape. Seong-Hwa Hur et al [11] simulated the buckling phenomenon of moderately thick-walled filament-wound carbon-epoxy composite cylinders under external hydrostatic pressure for underwater vehicle applications. All the analyses and test clearly proved that the cylinders collapse after buckling as they do not recover initial pressure. Major failure modes in these testes were related to the helical winding angles.

In general, a cylinder-type structure under external pressure loses its structural stiffness and shows a large amount of deflection at the buckling point. In some cases, the pressure-deflection curve shows a sharp drop after buckling in what has been termed unstable buckling. In such a structure, buckling directly leads to structural or partial failure. Therefore, buckling is considered as a critical design condition for the structure.

Although several authors reported evaluation of polymer composites for underwater vessels, comparative study of metallic alloys and polymer composites for specific operating conditions based on the geometrical details of working models is not reported. The main objective of this research was to evaluate the performance of glass/ carbon fibre reinforced epoxy along with the metallic alloys which are presently employed for underwater vessels by numerical approach.

\section{Finite Element Buckling Analysis}

Cylindrical shell of underwater vessels considered for the analysis is characterized by its overall length $\mathrm{L}$, inside diameter $\mathrm{D}$ and thickness $\mathrm{T}$. Critical external buckling pressure of vessels is denoted by Pcr. Material properties considered for the analysis are shown in Table 1 and Table 2 respectively. Finite Element Analysis software ANSYS was used to predict the buckling of the vessels. Cylindrical shell of underwater metallic vessels was analyzed by SHELL63 ANSYS element where isotropic material properties of elastic modulus and Poisson's ratio were taken into consideration. A Shell element has six degrees of freedom at each node. (i.e. Translations in the nodal $\mathrm{x}, \mathrm{y}$ and $\mathrm{z}$ directions and rotation about the nodal $\mathrm{x}, \mathrm{y}$ and $z$ axes), whereas fibre orientation, thickness distribution, stacking sequence and number of layers are the parameters used to examine the laminated shells by using linear layered structural shell element SHELL99. The element allows up to 250 different material layers with different orientations and orthotropic material properties in each of the layers. It has six degrees of freedom at each node.

Table 1. Material properties of Metallic structure

\begin{tabular}{l|c|c|c}
\hline \hline Material & Young's modulus (GPa) & Poisson's Ratio & Specific density \\
\hline High Strength Steel-HY 80 & 207 & 0.29 & 7.85 \\
\hline Aluminum Alloy & 70 & 0.33 & 2.7 \\
\hline Titanium Alloy & 119 & 0.32 & 4.4 \\
\hline \hline
\end{tabular}


Table: 1 Material properties of Composite structure

\begin{tabular}{|c|c|c|c|c|c|c|c|c|c|c|}
\hline Elastic Constant & $E_{1}$ & $\begin{array}{l}\mathrm{E}_{2} \\
\mathrm{GPa}\end{array}$ & $E_{3}$ & $\gamma_{12}$ & $\gamma_{23}$ & $\gamma_{13}$ & $\mathrm{G}_{12}$ & $\begin{array}{l}\mathrm{G}_{23} \\
\quad \mathrm{GPa}\end{array}$ & $\mathrm{G}_{13}$ & $\begin{array}{l}\text { Specific } \\
\text { Density }\end{array}$ \\
\hline Carbon/Epoxy & 156 & 9.56 & 6.57 & 0.27 & 0.492 & 0.34 & 5.47 & 2.8 & 3.92 & 2.1 \\
\hline Glass/Epoxy & 45.6 & 16.2 & 16.2 & 0.27 & 0.49 & 0.278 & 5.83 & 5.83 & 5.78 & 1.7 \\
\hline
\end{tabular}

Table: 3 Thickness of structure for various materials for CBP

\begin{tabular}{l|c}
\hline \hline Material & Thickness (mm) \\
\hline High Strength Steel (HY 80) & 6.75 \\
\hline Titanium Alloy & 8.25 \\
\hline Aluminum Alloy & 12 \\
\hline Carbon/epoxy composite & 15 \\
\hline Glass/epoxy composite & 18 \\
\hline \hline
\end{tabular}

\subsection{Linear buckling analysis}

Linear buckling analysis in ANSY is performed in two steps. First a static solution to the structure is obtained. In this analysis the Pre-buckling stress of the structure is calculated. The second step involves solving the eigenvalue problem given in the form of Eq. (1).This equation takes into consideration the Prebuckling stress effect matrix [S] calculated in the first step (ANSYS)

$\left([K]+\lambda_{i}[S]\right)\{\quad\}_{i}=\{0\}$

Where $[K]=$ Stiffness matrix

\section{$[S]=$ Stress stiffness matrix}

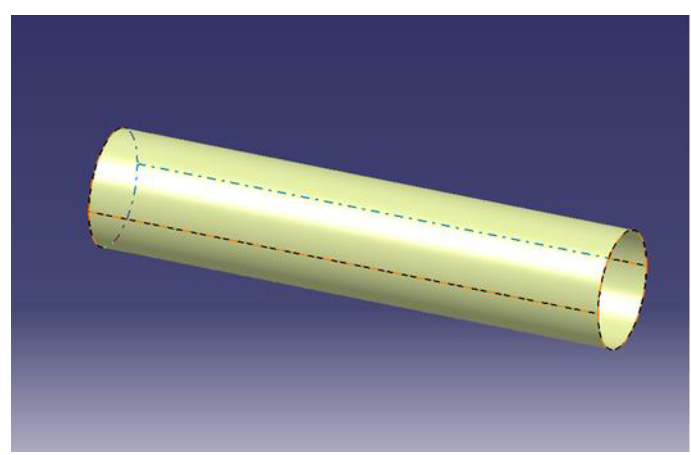

Fig. 1. Cylindrical shell Model of Underwater Vessel $\lambda_{i} \quad i^{\text {th }}$ eigenvalue (used to multiply the loads which generated [S])

$$
\left\{\quad i=\mathrm{i}^{\text {th }}\right. \text { eigenvector of displace- }
$$

ments

Once the Eigenvalues are found the critical buckling load is solved for $\mathrm{P}_{\mathrm{cr}}=\lambda^{*} \mathrm{P}_{\mathrm{a}}$

Where $\mathrm{P}_{\mathrm{cr}}$ are the critical buckling loads and $\mathrm{P}_{\mathrm{a}}$ are the applied loads.

Again usually only the lowest critical buckling load is of interests. The Block Lanczos method is chosen because it provides the most accuracy for the least computational time. The structure is modeled using ANSYS Software with inner diameter of $350 \mathrm{~mm}$ and length of $1650 \mathrm{~mm}$ as shown in Figure. 1 , the thickness of the structure was varied till the critical buckling pressure of $10 \mathrm{MPa}$ is reached for various materials.

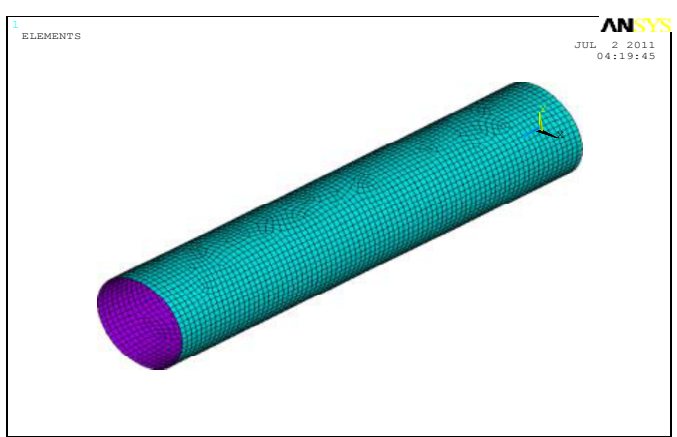

Fig. 2. Meshed model of Underwater Vesse 


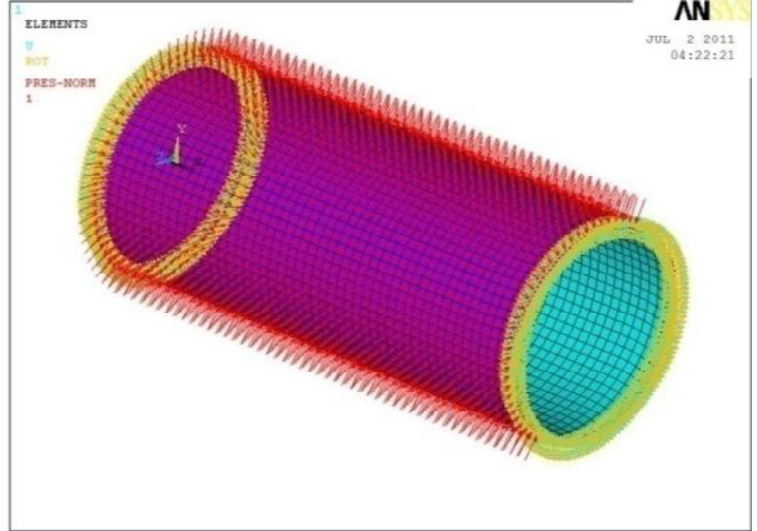

Fig. 3. Mesh model with Boundary Condition of Underwater Vessel

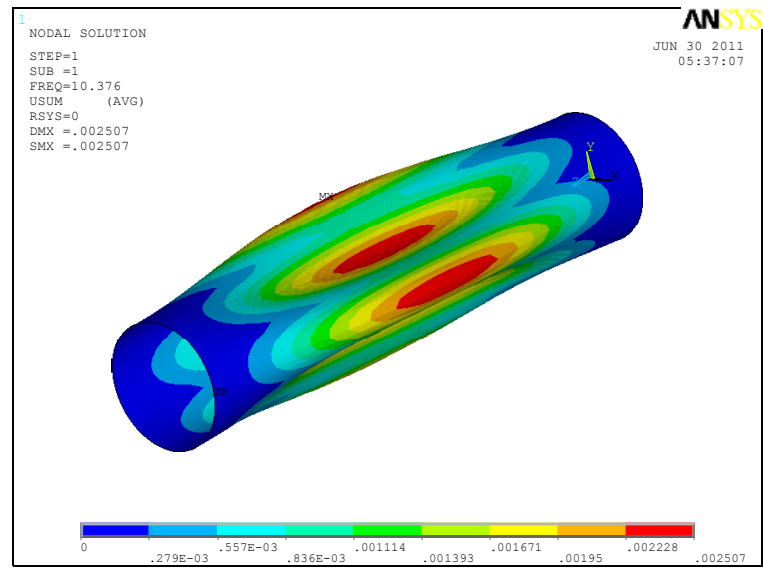

Fig. 5. Mode Shape for CBP of $10 \mathrm{MPa}$ for high strength steel

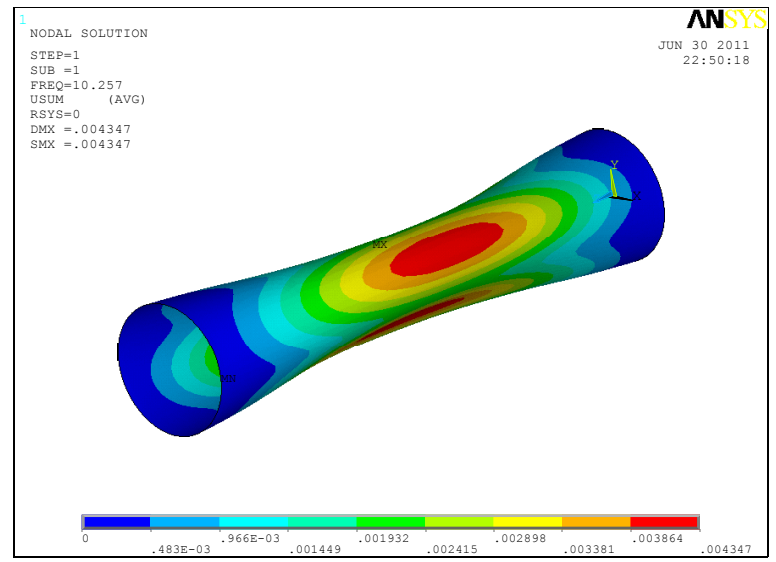

Fig. 7. Mode Shape for CBP of $10 \mathrm{MPa}$ for Glass/Epoxy

\subsection{Meshing of Models}

The model is meshed in ANSYS using free mesh using corresponding shell elements like SHELL63 and SHELL99 respectively as shown in Figure 2 and required quality parameter such as warpage,

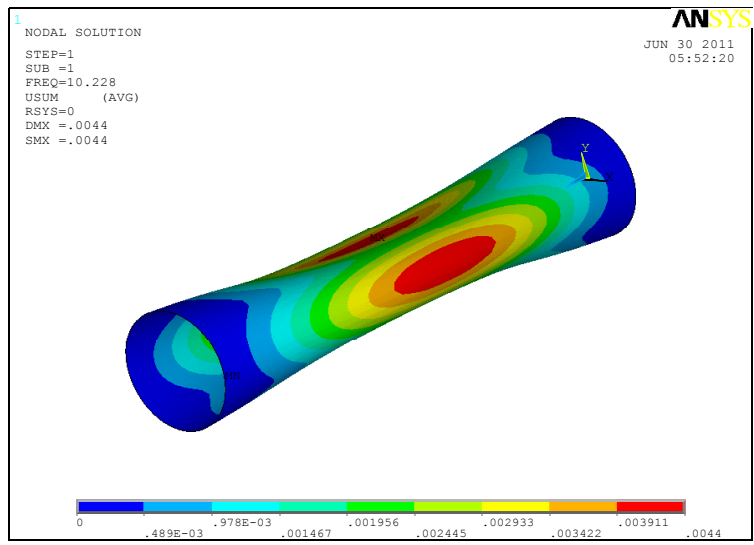

Fig. 4. Mode Shape for CBP of $10 \mathrm{MPa}$ for Aluminum Alloy Material

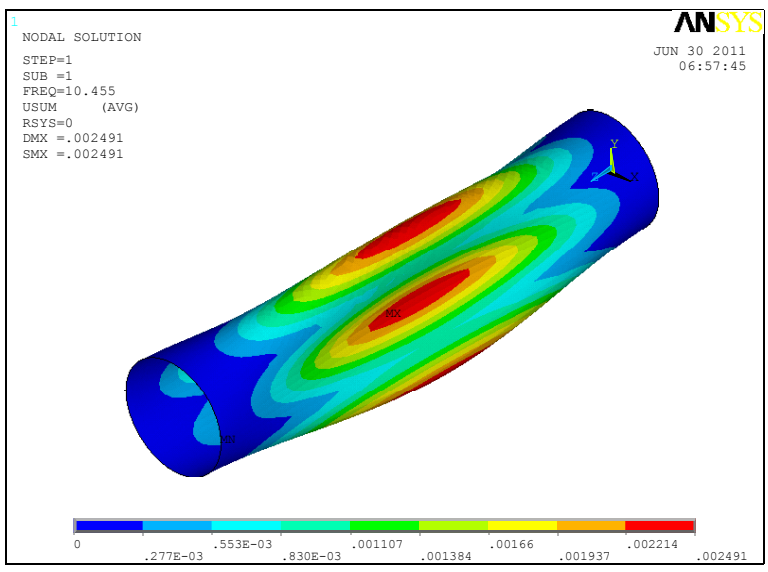

Fig. 6. Mode Shape for CBP of $10 \mathrm{MPa}$ for Titanium Alloy

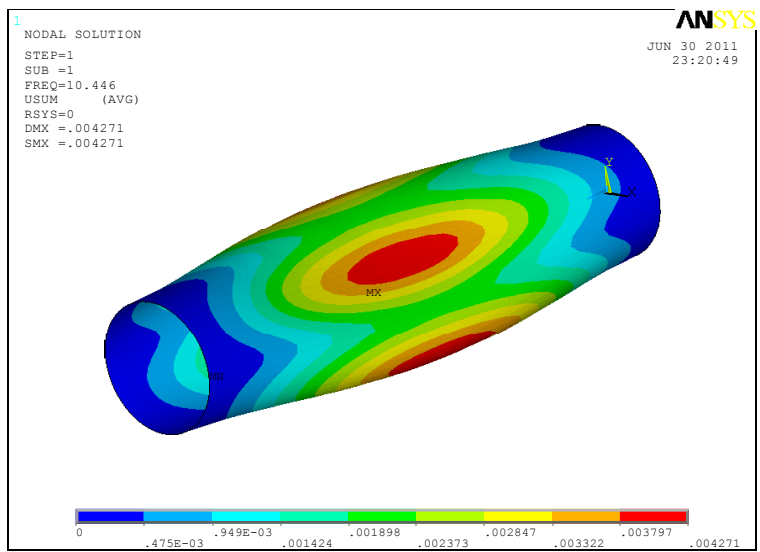

Fig. 8. Mode Shape for CBP of $10 \mathrm{MPa}$ for Carbon / epoxy

aspect ratio, skew angle, jacobian, minimum and maximum angle are satisfied.

\subsection{Boundary Conditions and External Pressure}

The boundary conditions for the finite element analysis are fixed along the both cylinder roots as 
shown in fig 3. An external pressure of $\mathrm{Pa}=1 \mathrm{MPa}$ is loaded on the external surfaces of composite layers. Static and buckling analyses are performed in sequence in order to obtain the critical buckling pressure Pcr.

\section{Results and Discussion}

Eigenvalues corresponding to Buckling modes are obtained from ANSYS output file and Critical buckling pressure is calculated for High Strength steel, Aluminum Alloy, Titanium Alloy, carbon/epoxy, glass/epoxy, different models of different thickness till the critical buckling pressure of $10 \mathrm{Mpa}$ is sustained. Figure 4 to 8 shows modes shape for metallic shells like High Strength Steel (HY 80), Aluminium Alloy, Titanium Alloy and polymer composite shell like Glass Epoxy and Carbon epoxy to withstand a critical buckling pressure of $10 \mathrm{MPa}$, thickness of these structures are tabulated in Table 3.

The thickness of composite structure was found higher than metallic for CBP of $10 \mathrm{MPa}$ as tabulated in Table 3 and shown in Figure 9 but due to its low density we see that weight of metallic structure is more than composite structure as shown in Figure 10. The results indicated weight savings of $46 \%$ in carbon/epoxy and $31 \%$ in glass / epoxy when compared with high strength steel, based on the thickness of the shell for sustaining $10 \mathrm{MPa}$ buckling pressure.

\section{Conclusions}

Based on the numerical analysis for buckling of underwater vessels for an operating depth of 1000 $\mathrm{m}$ the following conclusions were arrived: Carbon/epoxy employed for underwater vessels yields

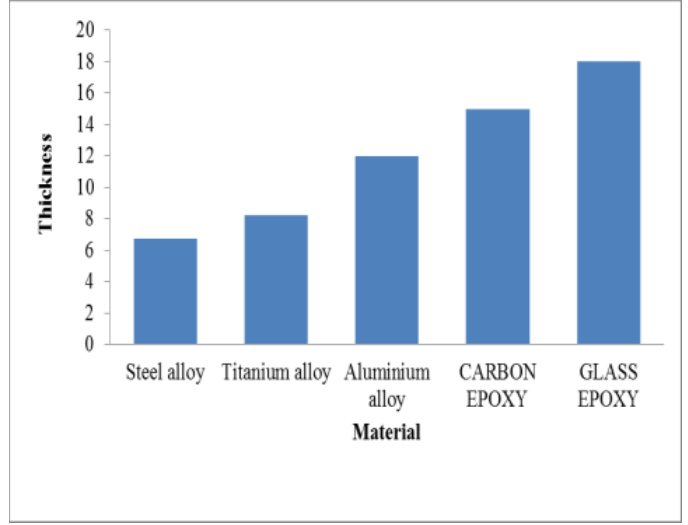

Fig. 1. Material verses thickness for Critical Buckling Pressure of $10 \mathrm{MPa}$ weight savings of $46 \%$ compared to high strength steel based on the thickness of the shell for sustaining $10 \mathrm{MPa}$ buckling pressure. Similar savings of $31 \%$ can be obtained by glass/epoxy instead of high strength steel.

\section{Conflict of Interest}

"The authors declare no conflict of interest".

\section{References}

[1] Ross CTF. A conceptual design of an underwater vehicle, Ocean Engineering, 33, 2006, 2087-2104.

[2] Joung T H, Lee C M, Hong S W, Kim J B, An $\mathrm{C} \mathrm{W}, A$ study on the results of the pressure vessel design. Structural analysis and pressure test of the semiautonomous underwater vehicle $(S A U V)$, Korean Society of Ocean Engineering, 18, 2004, 52-58.

[3] Carvelli V, Panzeri N, Poggi C. Buckling strength of GFRP under-water vehicles, Composites Part B, 32, 2001,89-101.

[4] Bisagni C. Dynamic buckling of fiber composite shells under impulsive axial compression, Thin-Walled Structure, 45, 2005, 499-514.

[5] Hilburger M W, Starnes Jr J H, Effect of imperfection of the buckling response of composite shells, Thin-Walled Structure, 42, 2004, 369397.

[6] Chryssanthopoulos M K, Poggi C, Probabilistic imperfection sensitivity analysis of axially compressed composite cylinders, Engineering Structure, 17(6), 1995, 398-406.

[7] Hahn H T, Jensen D W, Claus S J. Structural Design Criteria for filament wound composite shells, NASA CR195125, 1994.

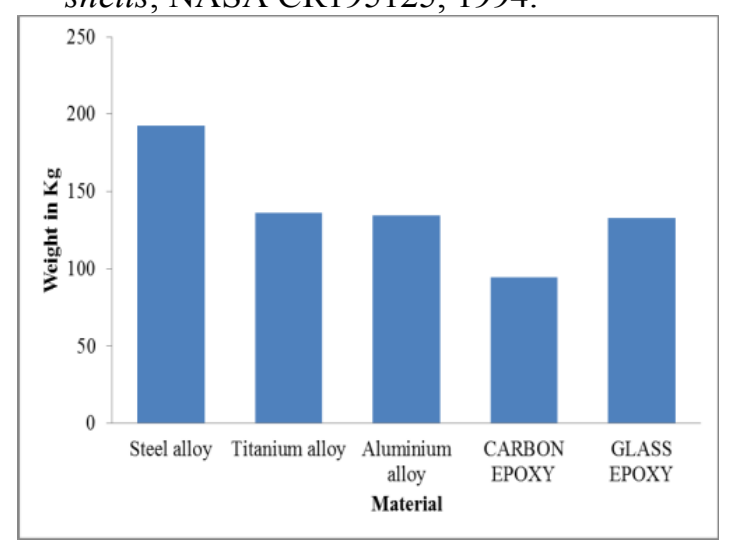

Fig. 10. Materials verses weight for Critical Buckling Pressure of $10 \mathrm{MPa}$ 
[8] Hernandez-Moreno H, Douchin B, Collombet F, Choqueuse D, Davies P, Influence of winding pattern on the mechanical behavior of filament wound composite cylinders under external pressure, Compos Sci Technol, 68(3-4), 2008, 1015-1024.

[9] Geier B, Meyer-Piening H-R, Zimmermann R. On the influence of laminate stacking on buckling of composite cylindrical shells subjected to axial compression, Composite Structure, 55, 2002, 467-474.

[10] Chul-Jin Moon, In-Hoon Kim, Bae-Hyeon Choi, Jin-Hwe Kweon, Jin-Ho Choi, Buckling of filament-wound composite cylinders subjected to hydrostatic pressure for underwater vehicle applications, Composite Structures, 90, 2010, 2241-2251.

[11] Seong-Hwa Hur, Hee-Jin Son, Jin-Hwe Kweon, Jin-Ho Choi, Postbuckling of composite cylinders under external hydrostatic pressure, Composite Structures, 86, 2008, 114-124

[12]Moorthy G, Narasimha Murthy H.N, Krishna M, Finite Element Analysis of Grid Stiffened composite structure of Underwater vehicle, International Journal of Advanced Engineering Research and Studies, 2, 2012, 151-153 